\title{
Logarithmic convexity of the eigenfunction of the Hessian operator in the ball
}

\author{
Yunhua Ye \\ School of Mathematics, Jiaying University, Meizhou, 514015, Guangdong Province, China \\ E-mail: mathyhye@163.com
}

Copyright (c)2015 Yunhua Ye. This is an open access article distributed under the Creative Commons Attribution License, which permits unrestricted use, distribution, and reproduction in any medium, provided the original work is properly cited.

\begin{abstract}
This paper is concerned with the logarithmic convexity of the eigenfunction of the Hessian operator. It is proved that the logarithm of the eigenfunction of the Hessian operator is strictly convex in the ball.
\end{abstract}

Keywords: eigenfunction; Hessian operator; logarithmic convexity; strict convexity.

\section{Introduction}

Convexity properties of solutions to partial differential equations are an interesting issue and have been investigated for a long time. One interesting question in this realm is the following: Is there a monotone real function $f$, such that $f(u(x))$ is concave or convex We recall here some results concerning this question. A classical example is that in 1971, Makar-Limanov [1] considered the following elliptic boundary value problem

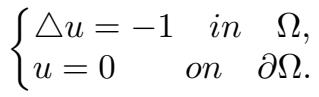

in a bounded and convex planar domain. By an ingenious argument involving the maximum principle, he proved that the square root $u^{\frac{1}{2}}$ of the solution $u$ is strictly concave. Another well-known example is that in 1976, Brascamp-Lieb [2] used a probabilistic approach to establish the log-concavity of the fundamental solution of diffusion equation with convex potential in a bounded and convex domain in $\mathbb{R}^{n}$. Consequently, they proved the log-concavity of the first eigenfunction of Laplacian equation in convex domain.

Since logarithmic convexity holds for the first eigenfunction of the Laplace operator, people naturally asks the question whether the same kind phenomena appears for the eigenfunctions of fully nonlinear operators. For the Monge-Ampère operator, the solutions are naturally convex. However, for the logarithmic convexity of the Hessian operator, there are only some works when the dimension of the domain is 3 . One interesting and typical result is that in 2010, Liu-Ma-Xu [3] considered the following eigenvalue problem for Hessian operator in a bounded and strictly convex domain

$\left\{\begin{array}{cll}\sigma_{2}\left(D^{2} u\right)=\lambda(-u)^{2} & \text { in } & \Omega \subset \mathbb{R}^{3} \\ u=0 & \text { on } & \partial \Omega\end{array}\right.$

where and in the following, $D^{2} u$ means the Hessian matrix of $u$ and $\sigma_{2}\left(D^{2} u\right)$ denotes the Hessian operator which is exactly the second elementary symmetric function of the eigenvalues of $D^{2} u$. They obtained the strict logarithmic 
concavity of the first eigenfunction. As an application, they get Brunn-Minkowski inequality for the Hessian eigenvalue and characterize the equality case.

It is an open question whether the logarithmic convexity property holds for the general Hessian operator $\sigma_{k}$ and for the domain being in general dimensions. We will answer this question when the domain is the ball $B_{R}(o) \subset \mathbb{R}^{n}$. We believe that these results remain true for the general convex domain in $\mathbb{R}^{n}$ and can try to solve these open question by using the macroscopic and microscopic methods.

In this paper, we consider the strict logarithmic convexity property of the admissible solutions of the following Hessian eigenfunctions problems in the ball $B_{R}(o) \subset \mathbb{R}^{n}$ :

$$
\left\{\begin{array}{rlrl}
\sigma_{k}\left(D^{2} u\right) & =\lambda(-u)^{k} & \text { in } & B_{R}(o) \subset \mathbb{R}^{n}, \\
u & <0 & \text { on } & B_{R}(o), \\
u & =0 \quad \text { on } & \partial B_{R}(o),
\end{array}\right.
$$

where $1 \leq k \leq n$.

For this eigenfunction, it has been proved in [4] that for $1<k<n$, up to a positive factor, for equation(1), there exist a unique negative admissible solution $u \in C^{\infty}(\Omega) \cap C^{1,1}(\bar{\Omega})$. Furthermore, equation (1) exists exactly one positive eigenvalue in a convex domain with smooth boundary. In this paper, we use Wang's results to deal with the case when the convex domain $\Omega$ are n-dimensional ball $B_{R}(o)$.

Our main results are stated as follows.

Theorem 1.1 Let $B_{R}(o)$ be the ball in $\mathbb{R}^{n}$ with radius $R>0$ and origin o. Let $u \in C^{\infty}\left(B_{R}(o)\right) \bigcap C^{1,1}\left(\overline{B_{R}}(o)\right)$ be the solutions to the Hessian eigenvalue problems of (1). Then $v=-\log (-u)$ is a strictly convex function in $B_{R}(o)$.

In the following sections, we will use two different methods to prove Theorem 1.1. In section 2, we will use differential approach. In section 3, we will use integral approach. We will use some facts concerning the properties of elementary symmetric function during the proving process. The definitions and the proof of these properties are standard, the readers can consult them on other reference books, such as [5] or [6], etc..

\section{Proof of Theorem 1.1 by differential approach}

In this section, we use differential method to prove the logarithmic convexity of the eigenfunctions of the Hessian operator $\sigma_{k}$ in the ball $B_{R}(o)$.

Since the domain is the ball $B_{R}(o)$ with radius $R$ and center $o$, then up to multiplying a constant, the problem (1) has a unique negative admissible solution $u \in C^{\infty}\left(B_{R}\right) \bigcap C^{1,1}\left(\overline{B_{R}}\right)$ and this unique solution is radially symmetric.

Therefore, we may assume the solution has the form

$u(x)=\phi(|x|)=\phi(r), \quad$ for $\quad|x|=r$,

where $r \in[0, R]$ and we see that $\phi(r)<0$ for $r \in[0, R]$. Then $\phi$ is an increasing function in $(0, R)$ and satisfies $\phi^{\prime}(0)=\phi(R)=0$.

For $1 \leq i, j \leq n$, a calculation shows that

$$
\begin{aligned}
\frac{\partial r}{\partial x_{i}} & =\frac{x_{i}}{r} \\
\frac{\partial^{2} r}{\partial x_{i} \partial x_{j}} & =-r^{-3} x_{i} x_{j}+r^{-1} \delta_{i j} .
\end{aligned}
$$

Then it follows that

$u_{i j}=\left(\phi^{\prime \prime} r^{-2}-\phi^{\prime} r^{-3}\right) x_{i} x_{j}+\phi^{\prime} r^{-1} \delta_{i j}$

Using the fact that the determinant of the matrix $\left\{A x_{i} x_{j}+B \delta_{i j}\right\}_{1 \leq i, j \leq k}$ is $A B^{k-1}|x|^{2}+B^{k}$, we have the radial form of the $\sigma_{k}$ operator

$\sigma_{k}\left(D^{2} u\right)=C_{n-1}^{k-1} \phi^{\prime k-1} \phi^{\prime \prime} r^{-k+1}+C_{n-1}^{k}\left(\phi^{\prime}\right)^{k} r^{-k}$,

where $C_{n-1}^{k}$ and $C_{n-1}^{k-1}$ are the combinatorial constants. 
Since $v=-\log (-\phi)$, we have

$$
\begin{aligned}
\phi^{\prime} & =e^{-v} v^{\prime} \\
\phi^{\prime \prime} & =e^{-v}\left(v^{\prime \prime}-v^{2}\right) .
\end{aligned}
$$

Substituting (2) and (3) into the equation (1), we obtain

$C_{n-1}^{k-1} r v^{\prime k-1} v^{\prime \prime}-C_{n-1}^{k-1} r v^{\prime k+1}+C_{n-1}^{k} v^{\prime k}=\lambda r^{k} \quad$ for $\quad 0<r<R$.

with conditions that

$v^{\prime}(0)=0 \quad$ and $\quad v^{\prime}(r)>0 \quad$ for $\quad 0<r<R$.

From the above, we know that

$v^{\prime \prime}(0) \geq 0$.

To prove Theorem 1.1, we first prove that $v$ is strictly convex in a neighborhood of the origin, we need to get a positive lower bound estimate of $v^{\prime \prime}(0)$. We divide $r^{k}$ on the both sides of equation (4) to obtain that

$C_{n-1}^{k-1} v^{\prime \prime}\left(\frac{v^{\prime}}{r}\right)^{k-1}-C_{n-1}^{k-1}\left(\frac{v^{\prime}}{r}\right)^{k-1} v^{\prime 2}+C_{n-1}^{k}\left(\frac{v^{\prime}}{r}\right)^{k}=\lambda$.

Letting $r \rightarrow 0$ in (5), we have

$C_{n}^{k} v^{\prime \prime}(0)^{k}=\lambda$,

where we have used the combinatorial identity $C_{n-1}^{k-1}+C_{n-1}^{k}=C_{n}^{k}$.

Therefore

$v^{\prime \prime}(0)=\sqrt[k]{\frac{\lambda}{C_{n}^{k}}}>0$

and $v^{\prime \prime}(r)$ is strictly positive in some neighborhood of the origin by smoothness of the solution.

We use a contradiction argument to show that $v(r)$ is strictly convex in $r \in[0, R]$. If not, there exists a smallest positive $r_{0}$ such that $v^{\prime \prime}\left(r_{0}\right)=0$. On one hand, we know $v^{\prime}(r)>0$ for $0<r \leq R$ and $v^{\prime \prime}(r)>0$ for $0<r<r_{0}$ and therefore

$v^{\prime \prime \prime}\left(r_{0}\right) \leq 0$.

On the other hand, we differentiate the equation (4) with respect to $r$ and evaluate at $r=r_{0}$ to obtain

$C_{n-1}^{k-1} r_{0} v^{\prime}\left(r_{0}\right)^{k-1} v^{\prime \prime \prime}\left(r_{0}\right)-C_{n-1}^{k-1} v^{\prime}\left(r_{0}\right)^{k+1}=\lambda k r_{0}^{k-1}$.

From the above, we deduce that

$v^{\prime \prime \prime}\left(r_{0}\right)=\frac{\lambda k}{C_{n-1}^{k-1}} \cdot \frac{r_{0}^{k-2}}{v^{\prime}\left(r_{0}\right)^{k-1}}+\frac{v^{\prime}\left(r_{0}\right)^{2}}{r_{0}}>0$.

A contradiction follows from (6) and (7) which tell us that $v^{\prime \prime}(r)>0$ in $[0, R)$. Therefore $v=-\log (-u)$ is strictly convex in $B_{R}(o)$.

\section{Proof of Theorem 1.1 by integral approach}

In this section, we use integral method to reprove Theorem 1.1.

We rewrite the equation (4) of section 2 into the following form

$C_{n-1}^{k-1}\left(\frac{v^{\prime}}{r}\right)^{k-1} v^{\prime \prime}+C_{n-1}^{k}\left(\frac{v^{\prime}}{r}\right)^{k}=\lambda+C_{n-1}^{k-1}\left(\frac{v^{\prime}}{r}\right)^{k-1} v^{\prime 2}$, 
which is equivalent to

$\frac{d}{d r}\left(r^{n-k} v^{\prime k}\right)=\frac{k r^{n-1}}{C_{n-1}^{k-1}}\left[\lambda+C_{n-1}^{k-1}\left(\frac{v^{\prime}}{r}\right)^{k-1} v^{\prime 2}\right], \quad$ for $\quad 0 \leq r<R$.

Integrating the above equation (8) from 0 to $r$, we get

$r^{n-k} v^{\prime}(r)^{k}=\frac{\lambda k}{n C_{n-1}^{k-1}} r^{n}+k \int_{0}^{r} v^{\prime}(s)^{k+1} s^{n-k} d s$.

Since $v^{\prime}>0$, solving the above equation, we obtain

$v^{\prime}(r)=\sqrt[k]{\frac{\lambda k}{n C_{n-1}^{k-1}} r^{k}+\frac{k}{r^{n-k}} \int_{0}^{r} v^{\prime}(s)^{k+1} s^{n-k} d s}$.

Taking the derivative with respect to $r$ in equation (9), we get

$$
\begin{aligned}
v^{\prime \prime}(r)= & \frac{\frac{\lambda k}{n C_{n-1}^{k-1}} r^{k-1}+v^{\prime}(r)^{k+1}-\frac{n-k}{r^{n+1-k}} \int_{0}^{r} v^{\prime}(s)^{k+1} s^{n-k} d s}{\left(\frac{\lambda k}{n C_{n-1}^{k-1}} r^{k}+\frac{k}{r^{n-k}} \int_{0}^{r} v^{\prime}(s)^{k+1} s^{n-k} d s\right)^{\frac{k-1}{k}}} \\
= & \frac{\frac{\lambda k}{n C_{n-1}^{k-1}}+\left[\frac{v^{\prime}(r)}{r}\right]^{k-1} v^{\prime}(r)^{2}-\frac{n-k}{r^{n}} \int_{0}^{r} v^{\prime}(s)^{k+1} s^{n-k} d s}{\left(\frac{\lambda k}{n C_{n-1}^{k-1}}+\frac{k}{r^{n}} \int_{0}^{r} v^{\prime}(s)^{k+1} s^{n-k} d s\right)^{\frac{k-1}{k}}} .
\end{aligned}
$$

To prove $v$ is strictly convex, we need to prove $v^{\prime \prime}(r)>0$ for $0 \leq r<R$.

Since $v^{\prime}(r)>0$ and $v^{\prime}(0)=0$, it is easy to see that $v^{\prime \prime}(0) \geq 0$. We then prove $v^{\prime \prime}(r)>0$ holds at the origin. We analyze the integral term in (10). By L'Hospital rule, we have

$$
\begin{aligned}
& \lim _{r \rightarrow 0} \frac{1}{r^{n}} \int_{0}^{r} v^{\prime}(s)^{k+1} s^{n-k} d s=\lim _{r \rightarrow 0} \frac{v^{\prime}(r)^{k+1} r^{n-k}}{n r^{n-1}} \\
= & \lim _{r \rightarrow 0} \frac{v^{\prime}(r)^{k+1}}{n r^{k-1}}=\frac{1}{n}\left(\lim _{r \rightarrow 0} \frac{v^{\prime}(r)}{r}\right)^{k-1} \lim _{r \rightarrow 0} v^{\prime}(r)^{2}=\frac{1}{n} v^{\prime \prime}(0)^{k-1} v^{\prime}(0)^{2}=0,
\end{aligned}
$$

where we used the fact $v^{\prime}(0)=0$.

Therefore from (10) and by letting $r \rightarrow 0$, we obtain

$v^{\prime \prime}(0)=\left[\frac{\lambda k}{n C_{n-1}^{k-1}}\right]^{\frac{1}{k}}>0$.

By smoothness of the solution, we know that $v^{\prime \prime}(r)>0$ in a neighborhood of the origin. In the following, we will prove $v^{\prime \prime}(r)$ will not change sign in the interval $(0, \mathrm{R})$, then we finish the proof of $v^{\prime \prime}(r)>0$ in $[0, R)$. We will use a contradiction argument. We assume the set $S=\left\{r \mid v^{\prime \prime}(s)>0, \forall s \in(0, r)\right\}$. The set $S$ is nonempty since it contains at least a neighborhood of the origin.

Let $\bar{r}=\sup S$, then we know $0<\bar{r} \leq R$ and $v^{\prime \prime}(r)>0$ in $[0, \bar{r})$. We also know

$v^{\prime}(\bar{r})>v^{\prime}(s)$ for $s<\bar{r}$.

We now prove $\bar{r}=R$. Indeed, if we assume on the contrary that $\bar{r}<R$. On one hand, by the definition of $\bar{r}$, we know

$v^{\prime \prime}(\bar{r})=0$.

On the other hand, by equation (10), we have

$v^{\prime \prime}(\bar{r})=\frac{\frac{\lambda k}{n C_{n-1}^{k-1}}+\left[\frac{v^{\prime}(\bar{r})}{\bar{r}}\right]^{k-1} v^{\prime}(\bar{r})^{2}-\frac{n-k}{\bar{r}^{n}} \int_{0}^{\bar{r}} v^{\prime}(s)^{k+1} s^{n-k} d s}{\left(\frac{\lambda k}{n C_{n-1}^{k-1}}+\frac{k}{\bar{r}^{n}} \int_{0}^{\bar{r}} v^{\prime}(s)^{k+1} s^{n-k} d s\right)^{\frac{k-1}{k}}}$ 
Noting that

$$
\begin{aligned}
& {\left[\frac{v^{\prime}(\bar{r})}{\bar{r}}\right]^{k-1} v^{\prime}(\bar{r})^{2}-\frac{n-k}{\bar{r}^{n}} \int_{0}^{\bar{r}} v^{\prime}(s)^{k+1} s^{n-k} d s } \\
= & \frac{\int_{0}^{\bar{r}}\left[(n-k+1) v^{\prime}(\bar{r})^{k+1}-(n-k) v^{\prime}(s)^{k+1}\right] s^{n-k} d s}{\bar{r}^{n}} \\
> & \frac{(n-k) \int_{0}^{\bar{r}}\left[v^{\prime}(\bar{r})^{k+1}-v^{\prime}(s)^{k+1}\right] s^{n-k} d s}{\bar{r}^{n}} \geq 0,
\end{aligned}
$$

where we have used (11).

Combining (13) and (14), we conclude that $v^{\prime \prime}(\bar{r})>0$, which is a contradiction with (12). Therefore $\bar{r}=R$. Consequently, $v$ is strictly convex for all $r \in[0, R)$ and Theorem 1.1 is proven again.

\section{Conclusion}

In this paper, we consider logarithmic convexity of the eigenfunction of the Hessian operator in the $n$-dimensional ball. For the corresponding result in general domain, we meet some technical difficulties, but we believe that the same kind result still holds true. These kind of convexities are of great interest in the study of fully-nonlinear elliptic equations. Finding their various applications of the convexities will help us understanding of the geometry of the solution surfaces.

\section{References}

[1] L.G. Makar-Limanov, Solution of Dirichlet's problem for the equation $\Delta u=-1$ on a convex region, Math. Notes Acad. Sci. USSR ,No.9, (1971), pp.52-53.

[2] H.J. Brascamp and E.H. Lieb, On extensions of the Brunn-Minkowski and Prekopa-Leindler theorems, including inequalities for log-concave functions, with an application to the diffusion equation, J. Func.Anal., No.22, (1976), pp.366-389.

[3] P.Liu, X.N.Ma and L.Xu, A Brunn-Minkowski inequatlity for the Hessian eigenvalue in three-dimensional convex domain, Adv.Math., No.225, (2010), pp.1616-1633.

[4] X.J.Wang, A class of fully nonlinear elliptic equations and related functionals, Indiana Univ. Math. J., No.43, (1994), pp.25-54.

[5] G.Huisken, C.Sinestrari, Convexity estimates for mean curvature flow and singularities of mean convex surfaces, Acta Math.,183 (1999), No. 1, pp.45-70.

[6] R. C. Reilly, On the Hessian of a function and the curvatures of its graph, Michigan Math. J., No.20,(1973), pp.373-383. 